\title{
THE SEARCH FOR NON-SYMMETRIC RIBBON KNOTS
}

\author{
CHRISTOPH LAMM
}

\begin{abstract}
We present the results of Axel Seeliger's tabulation of symmetric union presentations for ribbon knots with crossing numbers 11 and 12 and exhibit possible examples for ribbon knots which are not representable as symmetric unions. In addition, we give a complete atlas of band diagrams for prime ribbon knots with 11 and 12 crossings.
\end{abstract}

\section{INTRODUCTION AND OUTLINE OF RESULTS}

1.1. The setting. We recall that a knot $K \subset \mathbb{R}^{3}$ is a ribbon knot if it bounds a smoothly immersed disk $\mathbb{D}^{2} \uparrow \mathbb{R}^{3}$ whose only singularities are ribbon singularities, two sheets intersecting in an arc whose preimage consists of a properly embedded arc in $\mathbb{D}^{2}$ and an embedded arc interior to $\mathbb{D}^{2}$. Ribbon knots are frequently illustrated using the connected sum of a knot $K$ and its mirror image with reversed orientation, $-K$. The notion of symmetric union, introduced in 1957 by Shin'ichi Kinoshita and Hidetaka Terasaka [Kinoshita and Terasaka 57], generalises this by inserting crossings on the symmetry axis. The construction of a band with ribbon singularities remains the same as for $K \#-K$, with additional half-twists on the axis for each inserted crossing, see Figure 11. This article analyses the question which ribbon knots can be represented by symmetric unions - we call them symmetric ribbon knots - and which are non-symmetric. Currently the question whether non-symmetric ribbon knots exist is open.
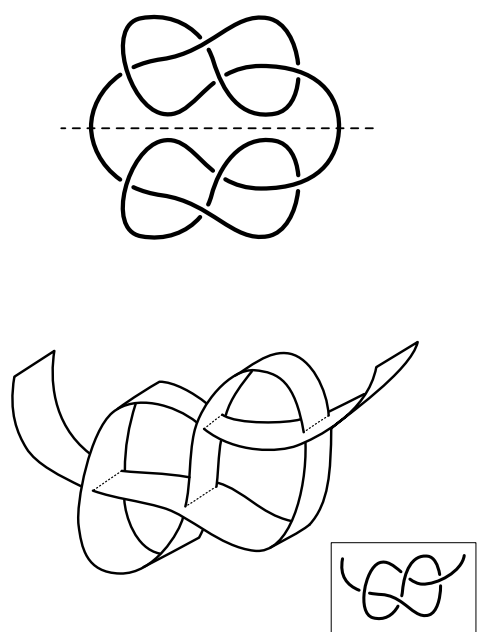
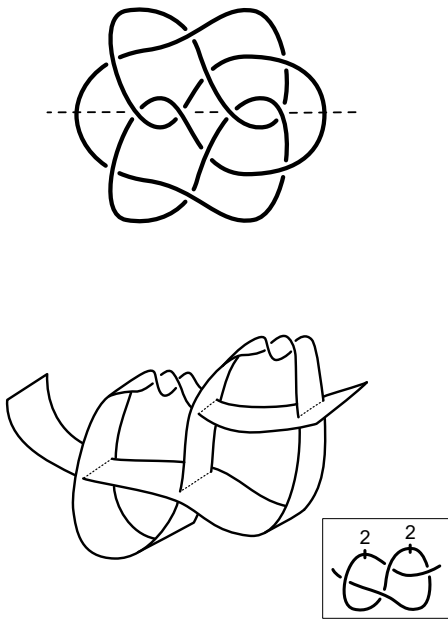

FIGURE 1. Symmetric unions and ribbons for the Kanenobu family of knots [Kanenobu 86], based on a diagram of $4_{1} \#-4_{1}$. On the right, the knot $12 \mathrm{n} 838$ is obtained by inserting crossings on the axis, in this case resulting in two full twists of the band. The insets show an abbreviated notation.

2000 Mathematics Subject Classification. 57M25, 57M27.

Key words and phrases. symmetric union presentations of ribbon knots, atlas of ribbon knots. 
1.2. Knot tabulation and unknown values in the table of knots. The tabulation of prime knots to 16 crossings was completed 20 years ago [Hoste, Thistlethwaite and Weeks 98]. A systematic list of unknown values for knot invariants was initiated in 2005 by Jae Choon Cha and Charles Livingston [Cha and Livingston 14]. Input for ribbon knots came in 2006 from Jenelle McAtee, Alexander Stoimenov and the author and for prime knots with 11 and 12 crossings 19 cases remained open. In 2008 Chris Herald, Paul Kirk and Charles Livingston ruled out 16 of these and showed that 12 a990 is smoothly slice [Herald, Kirk and Livingston 10]; the two cases not yet settled were 11n34 and 12a631.

1.3. The search for symmetric unions. In 2006 symmetric union presentations were known for all 21 prime ribbon knots with 10 or fewer crossings and for all 2-bridge ribbon knots [Lamm 00], [Lamm 06]. In the article [Eisermann and Lamm 07] we proposed a search project in order to assess how many ribbon knots with crossing number $\geq 11$ are symmetric unions. This search was performed in 2012 by Axel Seeliger [Seeliger 14] for the range of crossing numbers 11 through 14 and unexpectedly produced a symmetric ribbon for $12 \mathrm{a} 631$. As a result, with the possible exception of $11 \mathrm{n} 34$, the list of prime ribbon (and smoothly slice) knots up to 12 crossings was complete. Recently, in 2018, the knot $11 \mathrm{n} 34$ was shown not to be smoothly slice [Piccirillo 18]. Seeliger's search showed that out of 137 prime ribbon knots with 11 and 12 crossings the surprisingly high number of 122 knots have symmetric union presentations. In this article the diagrams of all of these knots are shown in an efficient way so that only 28 diagram templates are necessary.

1.4. Non-symmetric ribbons. The usefulness of this list of diagrams is further enhanced by four non-symmetric ribbons which generate all prime ribbon knots for which a symmetric diagram was not found. These four non-symmetric ribbons are shown in Figures 5,7 and 8 They generate knots with determinants 9,81 and 225 and we discuss their properties with the goal to find candidates for prime or composite ribbon knots which do not allow a symmetric union presentation. The most promising candidates seem to be the composite ribbon knots $3_{1} \# 8_{10}$ and $3_{1} \# 8_{11}$.

The 28 diagram templates and 4 non-symmetric ribbons constitute a complete atlas of knot diagrams for ribbon knots with 11 and 12 crossings, showing the ribbon property. For many knots it is also the first time that ribbon diagrams are available at all: for them the information to be ribbon was the result of a computer search, entered into the database Knotinfo [Cha and Livingston] without publishing the diagram information.

1.5. Characterisation and equivalences of bands. In the last two sections, we characterise bands constructed from symmetric union diagrams and introduce the knotoid notation for symmetric ribbon disks.

We distinguish two equivalence relations for ribbon knots: a knot given by a specific ribbon can be transformed to a symmetric union in a way that the ribbon is respected, or to a symmetric union presentation with any ribbon. We give examples and conjectures containing both types of equivalences. However, this topic - requiring the definition of band moves, similar to Reidemeister moves for knots - is not pursued in depth.

\section{BACKGROUND ON SLICE KNOTS AND BAND-SUMS}

For the purpose of this article we mainly need the following property of symmetric unions: a knot with a symmetric union diagram obtained from $K \#-K$ is a ribbon knot with determinant equal to the square of the determinant of $K$. The ingredient $K$ is called the partial knot of the symmetric union diagram. As we usually do not need to distinguish $K$ and $-K$ as partial knots, we use the notation $K_{ \pm}$. Because of the important property of the determinants, our atlas of symmetric ribbon knots is organised by (square) determinants. We refer to the articles [Eisermann and Lamm 07] and [Eisermann and Lamm 11] for additional topics, for instance symmetrical equivalence and the two-variable Jones polynomial defined for symmetric union diagrams. 
2.1. Three equivalent definitions of ribbon knots. We defined ribbon knots as immersed disks with only ribbon singularities. Alternatively, ribbon knots may be defined using band-sums, a generalisation of the connected sum of knots, see [Kanenobu 10] for more details. To motivate the occurence of glueing in bands we need a digression on the definition of slice knots in the 4-dimensional setting.
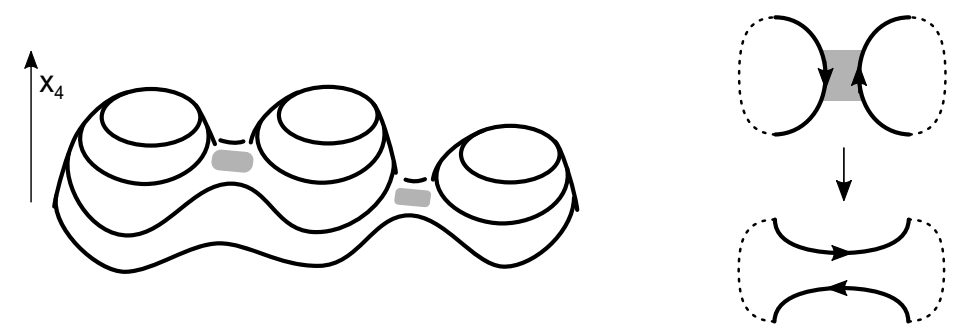

FIGURE 2. A disk with three local maxima and two saddles and the band-sum definition

Recall that a knot is smoothly slice if it is the boundary of a smooth disk $\mathbb{D}^{2}$ embedded in $\mathbb{R}_{+}^{4}=\left\{x \in \mathbb{R}^{4} \mid x_{4} \geq 0\right\}$. If the disk is given in Morse position, it can be described as a movie. Local maxima correspond to new trivial components, saddles to the insertion of bands (band-sums) and local minima to the disappearance of trivial components. Ribbon knots are characterised by disks without local minima. The slice-ribbon problem asks if every slice knot is a ribbon knot.

A band-sum in which the number of components of a link decreases by one (this is also called a 'fusion') is illustrated in the right of Figure 2.
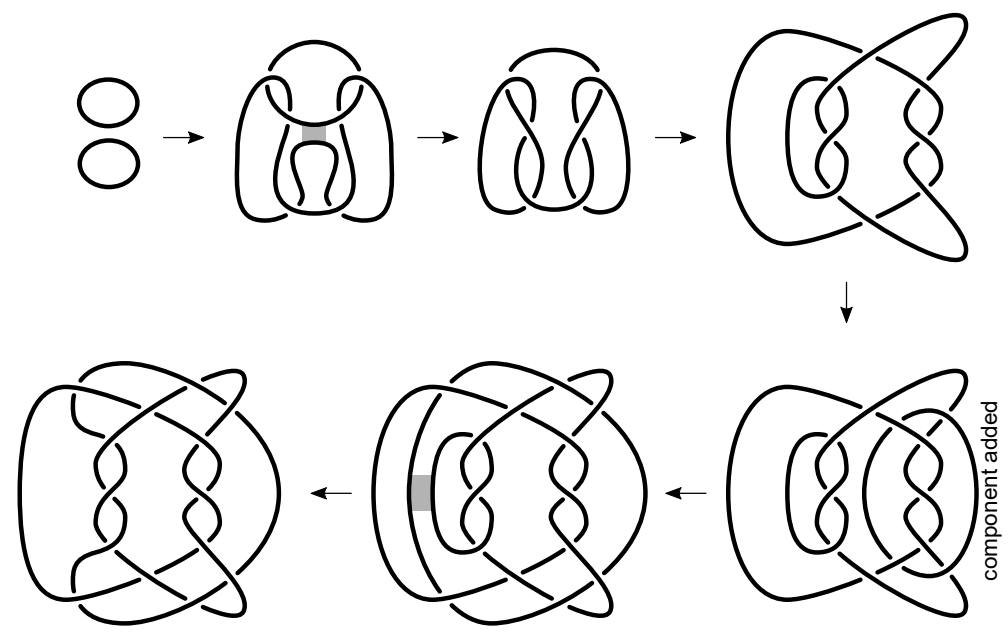

FIGURE 3. Example of a movie (for the knot 12a1225)

The insertion of bands to connect different components of a link occurs therefore in a natural way and by the arguments of the previous paragraph ribbon knots can be defined as knots obtained from a trivial $m+1$-component link by doing $m$ band-sum operations for some integer $m \geq 1$. In each operation the number of components decreases by one so that the result is a knot. As an example for $m=2$ we show a movie for the knot $12 \mathrm{a} 1225$ in Figure 3. It is based on a diagram in [Watson 07]. The two areas affected by band-sums are marked in grey. The (deformed) second band can be clearly seen in the diagram with ribbon singularities in the left of Figure 8 
2.2. Symmetric ribbon knots defined as symmetric band-sums of trivial links. We would like to apply the definition by band-sums to symmetric ribbon knots: in this case the trivial $m+1$-component link and the bands need to be symmetric to a plane; for the inserted bands we allow twists as in the definition of symmetric union diagrams. This definition with band-sums of a trivial link is equivalent to the usual definition of symmetric unions because the symmetric band can be cut open at the singularities and at the twist places, yielding a trivial link, and conversely bands can be glued in at the same places to obtain the symmetric band, see Figure 4 . This characterisation was also used by Toshifumi Tanaka in [Tanaka 10].

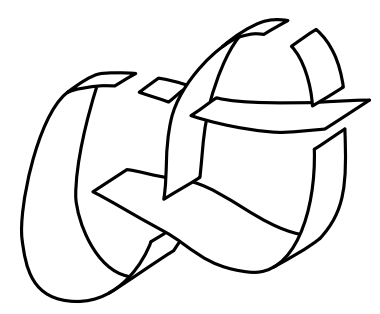

FIGURE 4. A trivial 6-component link which can be transformed into $12 \mathrm{n} 838$ by band-sums (compare with Figure 1). Untwisted bands are glued in where singularities have been cut out and twisted bands in the twist places.

We now analyse the symmetry in the movie in Figure 3 The first band-sum is applied to a symmetric trivial 2-component diagram and results in a symmetric union diagram for $3_{1} \#-3_{1}$. The third trivial component is added symmetrically with respect to a new axis perpendicular to the previous one and the second band-sum is again done symmetrically but with respect to the second axis. Of course, the fact that two different symmetry axes are used in the movie does not prove that the knot $12 \mathrm{a} 1225$ is a non-symmetric ribbon knot; it might possess a symmetric presentation as well.

A symmetry condition for bands with ribbon singularities due to Paolo Aceto is given in Section 5 .

\section{TABULATION OF SYMMETRIC RIBBON KNOTS WITH 11 AND 12 CROSSINGS}

3.1. Seeliger's tabulation method. In a computer search Seeliger used the Dowker-Thistlethwaite code to generate partial knots $K$, applying a new graph-theoretic method for testing realisability [Seeliger 14]. He then generated diagrams for $K \#-K$, allowing additional variants in cases where the Dowker-Thistlethwaite code is not unique (composite partial knots). After adding twist tangles on the symmetry axis, he checked the Jones polynomial of the symmetric union against the list of all Jones polynomials of prime ribbon knot candidates up to 14 crossings (defined as knots which satisfy the $f(t) \cdot f(1 / t)$ condition for the Alexander polynomial and having zero signature). Identification was done with Knotscape [Knotscape 99]. This resulted in a complete search for symmetric diagrams up to 16 crossings. Diagrams with more than 16 crossings were also generated, using heuristical strategies in order to find additional knots with low crossing number. Minimality of the crossing number is therefore assured also for 17 crossings. Once a knot had been found it was omitted from the search - we do not know if another diagram with the same number of crossings but e.g. different partial knot would have been found. For 11 and 12 crossing knots the result is summarised in the proposition:

Proposition 3.1 (Seeliger). Out of 137 prime ribbon knots with 11 and 12 crossing 122 knots have symmetric union presentations. 


\begin{tabular}{|c|c|c|c|c|c|c|c|c|c|c|c|}
\hline $11 \mathrm{a} 28$ & 121 & $\mathrm{~m}$ & $12 \mathrm{a} 100$ & 225 & sym & $12 \mathrm{a} 1029$ & 81 & $\mathrm{~m}$ & $12 \mathrm{n} 312$ & 49 & $\mathrm{~m}$ \\
\hline $11 \mathrm{a} 35$ & 121 & $\mathrm{~m}$ & $12 \mathrm{a} 173$ & 169 & $\mathrm{~m}$ & $12 \mathrm{a} 1034$ & 121 & $\mathrm{~m}$ & $12 \mathrm{n} 313$ & 1 & $\mathrm{~m}$ \\
\hline $11 \mathrm{a} 36$ & 121 & $\mathrm{~m}$ & $12 \mathrm{a} 183$ & 121 & $\mathrm{~m}$ & $12 \mathrm{a} 1083$ & 169 & sym & $12 \mathrm{n} 318$ & 1 & $\mathrm{~m}$ \\
\hline $11 \mathrm{a} 58$ & 81 & sym & $12 \mathrm{a} 189$ & 225 & sym & $12 \mathrm{a} 1087$ & 225 & sym & $12 \mathrm{n} 360$ & 49 & $\mathrm{~m}$ \\
\hline $11 \mathrm{a} 87$ & 121 & $\mathrm{~m}$ & $12 \mathrm{a} 211$ & 169 & $\mathrm{~m}$ & $12 \mathrm{a} 1105$ & 289 & $\mathrm{~m}$ & $12 \mathrm{n} 380$ & 81 & $\mathrm{~m}$ \\
\hline $11 \mathrm{a} 96$ & 121 & $\mathrm{~m}$ & $12 \mathrm{a} 221$ & 169 & $\mathrm{~m}$ & $12 \mathrm{a} 1119$ & 169 & $\mathrm{~m}$ & $12 \mathrm{n} 393$ & 49 & $\mathrm{~m}$ \\
\hline 11a103 & 81 & - & $12 \mathrm{a} 245$ & 225 & sym & $12 \mathrm{a} 1202$ & 169 & $\mathrm{~m}$ & 12 n394 & 25 & $\mathrm{~m}$ \\
\hline 11a115 & 121 & $\mathrm{~m}$ & $12 \mathrm{a} 258$ & 169 & $\mathrm{~m}$ & $12 \mathrm{a} 1225$ & 225 & - & 12 n397 & 49 & $\mathrm{~m}$ \\
\hline 11a164 & 169 & sym & $12 \mathrm{a} 279$ & 169 & $\mathrm{~m}$ & $12 \mathrm{a} 1269$ & 169 & $\mathrm{~m}$ & 12 n399 & 81 & $\mathrm{~m}$ \\
\hline 11a165 & 81 & - & $12 \mathrm{a} 348$ & 225 & - & $12 \mathrm{a} 1277$ & 121 & $\mathrm{~m}$ & $12 \mathrm{n} 414$ & 25 & $\mathrm{~m}$ \\
\hline 11a169 & 121 & $\mathrm{~m}$ & $12 \mathrm{a} 377$ & 225 & sym & $12 \mathrm{a} 1283$ & 81 & $\mathrm{~m}$ & $12 \mathrm{n} 420$ & 81 & $\mathrm{~m}$ \\
\hline $11 \mathrm{a} 201$ & 81 & - & $12 \mathrm{a} 425$ & 81 & $\mathrm{~m}$ & & & & $12 \mathrm{n} 430$ & 1 & $\mathrm{~m}$ \\
\hline $11 \mathrm{a} 316$ & 121 & $\mathrm{~m}$ & $12 \mathrm{a} 427$ & 225 & $\mathrm{~m}$ & $12 \mathrm{n} 4$ & 81 & $\mathrm{~m}$ & $12 \mathrm{n} 440$ & 81 & $\mathrm{~m}$ \\
\hline \multirow[t]{2}{*}{$11 \mathrm{a} 326$} & 169 & sym & $12 \mathrm{a} 435$ & 225 & sym & $12 \mathrm{n} 19$ & 1 & $\mathrm{~m}$ & $12 \mathrm{n} 462$ & 25 & $\mathrm{~m}$ \\
\hline & & & $12 \mathrm{a} 447$ & 121 & $\mathrm{~m}$ & $12 \mathrm{n} 23$ & 9 & $\mathrm{~m}$ & $12 \mathrm{n} 501$ & 49 & $\mathrm{~m}$ \\
\hline $11 n 4$ & 49 & $\mathrm{~m}$ & $12 \mathrm{a} 456$ & 225 & sym & $12 \mathrm{n} 24$ & 49 & $\mathrm{~m}$ & $12 \mathrm{n} 504$ & 121 & $\mathrm{~m}$ \\
\hline $11 \mathrm{n} 21$ & 49 & $\mathrm{~m}$ & $12 \mathrm{a} 458$ & 289 & sym & $12 \mathrm{n} 43$ & 81 & $\mathrm{~m}$ & $12 \mathrm{n} 553$ & 81 & $\mathrm{~m}$ \\
\hline $11 \mathrm{n} 37$ & 25 & $\mathrm{~m}$ & $12 \mathrm{a} 464$ & 225 & sym & $12 \mathrm{n} 48$ & 49 & $\mathrm{~m}$ & $12 \mathrm{n} 556$ & 81 & $\mathrm{~m}$ \\
\hline $11 \mathrm{n} 39$ & 25 & $\mathrm{~m}$ & $12 \mathrm{a} 473$ & 289 & sym & $12 \mathrm{n} 49$ & 81 & sym & $12 \mathrm{n} 582$ & 9 & $\mathrm{~m}$ \\
\hline $11 \mathrm{n} 42$ & 1 & $\mathrm{~m}$ & $12 \mathrm{a} 477$ & 169 & $\mathrm{~m}$ & $12 \mathrm{n} 51$ & 9 & - & $12 \mathrm{n} 605$ & 9 & $\mathrm{~m}$ \\
\hline $11 \mathrm{n} 49$ & 1 & $\mathrm{~m}$ & $12 \mathrm{a} 484$ & 289 & sym & $12 \mathrm{n} 56$ & 9 & - & $12 \mathrm{n} 636$ & 81 & $\mathrm{~m}$ \\
\hline $11 \mathrm{n} 50$ & 25 & $\mathrm{~m}$ & $12 \mathrm{a} 606$ & 169 & $\mathrm{~m}$ & $12 \mathrm{n} 57$ & 9 & - & $12 \mathrm{n} 657$ & 81 & $\mathrm{~m}$ \\
\hline $11 \mathrm{n} 67$ & 9 & - & $12 \mathrm{a} 631$ & 225 & sym & $12 \mathrm{n} 62$ & 81 & - & $12 \mathrm{n} 670$ & 25 & $\mathrm{~m}$ \\
\hline $11 \mathrm{n} 73$ & 9 & - & $12 \mathrm{a} 646$ & 169 & $\mathrm{~m}$ & $12 \mathrm{n} 66$ & 81 & - & $12 \mathrm{n} 676$ & 9 & sym \\
\hline $11 \mathrm{n} 74$ & 9 & - & $12 \mathrm{a} 667$ & 121 & $\mathrm{~m}$ & $12 \mathrm{n} 87$ & 49 & $\mathrm{~m}$ & $12 \mathrm{n} 702$ & 121 & $\mathrm{~m}$ \\
\hline $11 \mathrm{n} 83$ & 49 & $\mathrm{~m}$ & $12 \mathrm{a} 715$ & 169 & $\mathrm{~m}$ & $12 \mathrm{n} 106$ & 81 & $\mathrm{~m}$ & $12 \mathrm{n} 706$ & 49 & $\mathrm{~m}$ \\
\hline 11 n97 & 9 & - & $12 \mathrm{a} 786$ & 169 & sym & $12 \mathrm{n} 145$ & 25 & $\mathrm{~m}$ & $12 \mathrm{n} 708$ & 49 & $\mathrm{~m}$ \\
\hline $11 n 116$ & 1 & $\mathrm{~m}$ & $12 \mathrm{a} 819$ & 169 & $\mathrm{~m}$ & $12 \mathrm{n} 170$ & 81 & $\mathrm{~m}$ & $12 \mathrm{n} 721$ & 25 & $\mathrm{~m}$ \\
\hline $11 n 132$ & 25 & $\mathrm{~m}$ & $12 \mathrm{a} 879$ & 121 & $\mathrm{~m}$ & $12 \mathrm{n} 214$ & 1 & $\mathrm{~m}$ & $12 \mathrm{n} 768$ & 25 & $\mathrm{~m}$ \\
\hline $11 n 139$ & 9 & $\mathrm{~m}$ & $12 \mathrm{a} 887$ & 289 & sym & $12 \mathrm{n} 256$ & 25 & $\mathrm{~m}$ & $12 \mathrm{n} 782$ & 81 & $\mathrm{~m}$ \\
\hline \multirow[t]{2}{*}{$11 \mathrm{n} 172$} & 49 & $\mathrm{~m}$ & $12 \mathrm{a} 975$ & 225 & sym & $12 \mathrm{n} 257$ & 25 & $\mathrm{~m}$ & $12 \mathrm{n} 802$ & 121 & $\mathrm{~m}$ \\
\hline & & & $12 \mathrm{a} 979$ & 225 & sym & $12 \mathrm{n} 268$ & 9 & $\mathrm{~m}$ & $12 \mathrm{n} 817$ & 49 & $\mathrm{~m}$ \\
\hline $12 \mathrm{a} 3$ & 169 & sym & $12 \mathrm{a} 990$ & 225 & - & $12 \mathrm{n} 279$ & 25 & $\mathrm{~m}$ & 12 n838 & 25 & $\mathrm{~m}$ \\
\hline $12 \mathrm{a} 54$ & 169 & $\mathrm{~m}$ & $12 \mathrm{a} 1011$ & 121 & $\mathrm{~m}$ & $12 \mathrm{n} 288$ & 49 & $\mathrm{~m}$ & $12 \mathrm{n} 870$ & 25 & $\mathrm{~m}$ \\
\hline $12 \mathrm{a} 77$ & 225 & sym & $12 \mathrm{a} 1019$ & 361 & $\mathrm{~m}$ & 12 n309 & 1 & $\mathrm{~m}$ & $12 \mathrm{n} 876$ & 81 & $\mathrm{~m}$ \\
\hline
\end{tabular}

TABLE 1. A list of all 137 prime ribbon knots with crossing numbers 11 or 12 together with determinant and status information. Abbreviations: ' $\mathrm{m}$ ' = a minimal symmetric union diagram is known, 'sym' = a symmetric union diagram is known but is not necessarily minimal, ' - ' = no symmetric union diagram has been found.

The 'symmetry status' of the remaining 15 prime ribbon knots is currently unknown. We discuss their properties in Section 4

3.2. Finding a small number of diagram templates. To save space in the appendix of this article, we tried to describe as many of the 122 diagrams as possible in a template form. For instance, all 15 ribbon knots with partial knot $5_{2}$ can be described in a uniform way using a template with 3 twist parameters. In other cases several templates were needed and they are listed as 'version a', 'version b' (for the trivial partial knot and for $6_{1}, 6_{2}, 7_{3}$, 75 ) and, in addition, 'version c' for $3_{1}$. We show the templates with one choice of twist parameters and underline the corresponding knot in the annotating list. If a diagram is used only for one ribbon knot, then we do not depict it as a template with parameters but as an individual symmetric union diagram. See, for example, the diagram for $12 \mathrm{n} 706$ with determinant 49 in the appendix. The template parameters were checked with Knotscape to make sure that the knot types are correct. Note that partial knots and the number of 
inserted twists on the axis agree with Seeliger's list, but our diagrams need not be the same and may be symmetrically inequivalent.

An overview is given in Table 1 . It contains all 137 prime ribbon knots with 11 and 12 crossings, their determinant and a 'symmetry status' information.

As an example, assume that you are interested in alternating ribbon knots with 11 crossings and determinant 169. In the first column of Table 1 we find the two knots 11a164 and 11a326 with the information 'sym' meaning that for these knots a symmetric but not necessarily minimal diagram exists. They are found in the appendix for the 73 'version a'-template and for both knots 5 crossings are inserted on the axis, giving 19 crossings in total. Because the search was complete only through 16 crossings, the diagrams need not be minimal.

As a second example, we look at all non-alternating knots in Table 1 and observe that with the exception of two knots (12n49 and 12n676) all symmetric diagrams are minimal (they have 17 or less crossings). This is in contrast to alternating knots where many symmetric diagrams with 18 or more crossings occur.

3.3. Properties of the symmetric union presentations in the atlas. To give some orientation, we comment on the properties of the partial knots, the occurence of composite ribbon knots and of strongly-positive-amphichiral knots in the atlas:

A) The following properties have been observed with respect to the partial knots:

- (bridge number) Among the prime partial knots in the atlas only $8_{21}$ has bridge number greater than 2. Because all non-trivial prime knots with up to 7 crossings are two-bridge knots, this is not as surprising as one may think at first.

- (composite) There are symmetric unions with composite partial knots $3_{1} \# 3_{1}, 3_{1} \#-$ $3_{1}$ and $3_{1} \# 4_{1}$. These are interesting because if a symmetric union is constructed from a composite partial knot in another way it is itself composite.

- (non-alternating) There is only one non-alternating partial knot in the list - again $8_{21}$ - but for determinants 1 and 9 we have non-alternating diagrams of the trivial knot and of the trefoil. All other partial diagrams in the atlas are alternating.

B) We note a new phenomenon concerning composite ribbon knots: for crossing numbers $\leq 10$ composite ribbon knots are always of the form $K \#-K$ with prime $K$, but for crossing numbers 11 and 12 other types of composite ribbon knots exist: $3_{1} \# 8_{10}, 3_{1} \# 8_{11}$ and also $6{ }_{1} \# 3_{1} \#-33_{1}, 3_{1} \# 3_{1} \#-3_{1} \#-3_{1}$, see [Kearney 13], [Livingston 04]. Of course, in the cases $3_{1} \# 8_{10}$ and $3_{1} \# 8_{11}$ suitable mirror variants have to be chosen so that the total signature is zero. It is clear that $6_{1} \# 3_{1} \#-3_{1}$ and $3_{1} \# 3_{1} \#-3_{1} \#-3_{1}$ are symmetric because they are composed of symmetric ribbon knots. On the other hand, $3_{1} \# 8_{10}$ and $3_{1} \# 8_{11}$ are interesting candidates for non-symmetric ribbon knots.

C) A knot is called strongly-positive-amphichiral if it has a diagram which is mapped to its mirror image by a rotation of $\pi$, preserving the orientation.

Obviously, composite knots consisting of a knot and its mirror image are stronglypositive-amphichiral. Prime strongly-positive-amphichiral knots are rare, however: In [Boocher, Daigle, Hoste and Zheng 09], p. 482, it was stated that according to [Hoste, Thistlethwaite and Weeks 98] there are only three prime knots with 12 or fewer crossings that are strongly-positiveamphichiral: $10_{99}, 10_{123}$ and 12a427. Contrary to this, Seeliger's search produced diagrams for $12 \mathrm{a} 1202$ and $12 \mathrm{n} 706$ showing this symmetry. It occurs for a symmetric union diagram if in addition to its symmetry axis it possesses a rotational symmetry axis perpendicular to the plane, provided that the knot's orientation is preserved under rotation by $\pi$. We found such diagrams later also for $12 \mathrm{a} 1019$ and $12 \mathrm{a} 1105$ (shown in the appendix). Therefore we ask:

Question 3.2. Is the list of prime strongly-positive-amphichiral knots with at most 12 crossings, consisting of $10_{99}, 10_{123}, 12 \mathrm{a} 427,12 \mathrm{a} 1019,12 \mathrm{a} 1105,12 \mathrm{a} 1202$ and 12n706, complete? Using Knotinfo data, the only undecided case seems to be $12 \mathrm{a} 435$.

In the next section we analyse the 15 ribbon knots which were not found to be symmetric unions and try to find common properties which may lead to a topological explanation. 


\section{ANALYSING NON-SYMMETRIC RIBBONS}

The 15 prime ribbon knots for which a symmetric union presentation is missing have determinants 9 ( 7 cases), 81 ( 5 cases) and 225 ( 3 cases). For the cases with determinants 9 and 81 we found two non-symmetric ribbons (only two ribbons covering all 12 cases) suggesting that these knots indeed share common topological obstructions that prevent their presentation as symmetric unions. The 3 cases with determinant 225 are also covered by two ribbons.

4.1. A non-symmetric ribbon for the remaining ribbon knots with determinant 9. For the 7 knots with determinant 9 (i.e. $11 n 67,11 n 73,11 n 74,11 n 97,12 n 56$ and 12n57) we found the ribbon $\mathscr{A}$ shown in Figure 5 . The figure also shows an effort to symmetrise the diagram: We put the tangle with $T$ half-twists on the symmetry axis indicated by the tangle $3_{1} \#-3_{1}$ in the diagram's upper part. Then the $S$-tangle appears as a 'symmetry defect' on the left side. This indicates that for some values of $S$ it might be possible to obtain a symmetric diagram but for others it might not be possible.

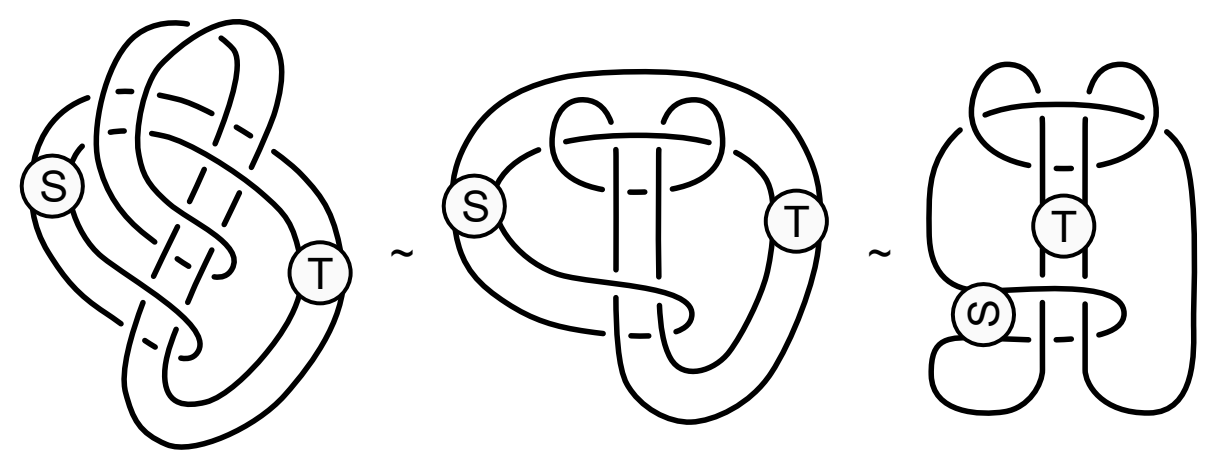

FIGURE 5. The ribbon $\mathscr{A}(S, T)$ and two transformations

Varying the twist parameters $S$ and $T$ we obtain the knots in Table 2 . For the values $S=-1,0$ they can indeed be presented as symmetric unions.

\begin{tabular}{|c|c|c|c|c|c|}
\hline $\mathrm{S} \backslash \mathrm{T}$ & -2 & -1 & 0 & 1 & 2 \\
\hline-4 & & $13 \mathrm{n} 1666$ & $13 \mathrm{n} 1313$ & $13 \mathrm{n} 1674$ & \\
-3 & & $12 \mathrm{n} 51$ & $12 \mathrm{n} 56$ & $12 \mathrm{n} 268$ & \\
-2 & $13 \mathrm{n} 612$ & $11 \mathrm{n} 67$ & $11 \mathrm{n} 74$ & $11 \mathrm{n} 97$ & $13 \mathrm{n} 973$ \\
\hline-1 & $10_{140}$ & $9_{46}$ & $8_{20}$ & $6_{1}$ & $3_{1} \#-3_{1}$ \\
0 & $3_{1} \#-3_{1}$ & $6_{1}$ & $8_{20}$ & $9_{46}$ & $10_{140}$ \\
\hline 1 & $13 \mathrm{n} 835$ & $11 \mathrm{n} 97$ & $11 \mathrm{n} 73$ & $11 \mathrm{n} 67$ & $13 \mathrm{n} 611$ \\
2 & & $12 \mathrm{n} 268$ & $12 \mathrm{n} 57$ & $12 \mathrm{n} 51$ & \\
3 & & $13 \mathrm{n} 1674$ & $13 \mathrm{n} 1316$ & $13 \mathrm{n} 1666$ & \\
\hline
\end{tabular}

TABLE 2. Overview of knot types for the ribbon $\mathscr{A}(S, T)$ with determinant 9 (knots and their mirror images are not distinguished)

With the exception of $12 \mathrm{n} 268$, for none of the other knots in the table was a symmetric diagram found. We discuss the knots in Table 2 in more detail: 
- The 11 and 12 crossings knots in the table (with the exception of 12n268) are the ribbon knots with determinant 9 for which no symmetric union presentation was found.

- For the knot 12 n268 a symmetric diagram is known, see template $3_{1}$, version b.

- For $S=0$ the ribbon can be symmetrised, as shown in Figure 6. The case $S=-1$ is similar and results in a total number of half-twists on the axis of $T-2$ instead of $T+2$. These knots are generated by template $3_{1}$, version a.

- In the column $T=0$ the knots $11 \mathrm{n} 73$ and $11 \mathrm{n} 74$ are mutants, the same is true for $12 \mathrm{n} 56$ and $12 \mathrm{n} 57$.

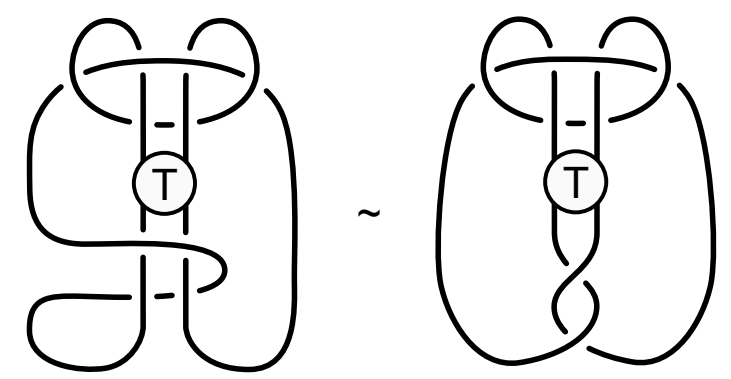

FIGURE 6. Symmetrising the ribbon $\mathscr{A}(S, T)$ in the case $S=0$

The observation about mutants can be generalised (if an unoriented knot diagram is obtained from $D$ by switching all crossings we denote it by $\left.D^{*}\right)$ :

Proposition 4.1. The knots $\mathscr{A}(-S,-T)$ and $\mathscr{A}(S-1, T)^{*}$ are mutants.

Proof. Let $S \geq 1, T \geq 0$. Start with $\mathscr{A}(-S,-T)$ and mirror the diagram shown on the right in Figure 5 in the plane. This almost yields $\mathscr{A}(S, T)$ : the upper part is exactly as in $\mathscr{A}(S, T)$ if it is mutated by a vertical flip. From the tangle with $S$ crossings in the lower part one half-twist is needed to put the overcrossing arc in the right position. This results in $\mathscr{A}(S-1, T)^{*}$.

In addition to the mutant pairs $(11 \mathrm{n} 73,11 \mathrm{n} 74)$ and $(12 \mathrm{n} 56,12 \mathrm{n} 57)$ this explains the pairs (13n611, 13n612), (13n835, 13n973) and (13n1313, 13n1316). For $T= \pm 1$, however, the mutation seems to preserve the knot types. Whether this is true for all $S$ is an open question.

We have the following conjecture about this family. It is supported also by the knots with 13 crossings in the table.

Conjecture 4.2. With the exception of $12 \mathrm{n} 268$ and the knots with $S=-1,0$, the knots $\mathscr{A}(S, T)$ do not have symmetric union presentations.

4.2. A non-symmetric ribbon for the remaining ribbon knots with determinant 81 . For the 5 knots with determinant 81 (i.e. 11a103, 11a165, 11a201, 12n62 and 12n66) we found the ribbon $\mathscr{B}$ shown in Figure 7 A transformation to a diagram with more symmetry is possible as well and we also detect a 'symmetry defect'. (By this we mean the mixed occurence of symmetry and its violation, similar to the case in Figure 3 . Proposition 5.1 contains a rigorous characterisation of non-symmetric bands.)

Varying the twist parameters $R, S$ and $T$ we obtain the knots in Table 3 . The left column contains the 5 knots with 11 and 12 crossings which are candidates for non-symmetric ribbon knots. In the middle column we show, in addition, knots with 13 crossings for which no symmetric presentation is known and the right column contains ribbon knots with known symmetric union presentations. 

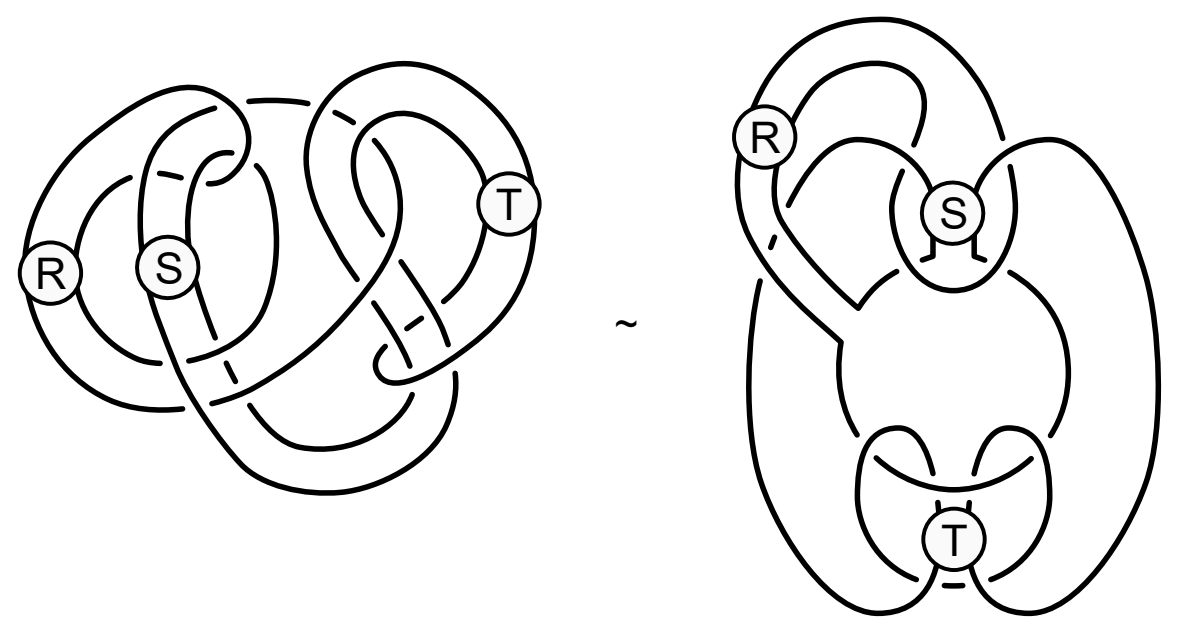

FIGURE 7. The ribbon $\mathscr{B}(R, S, T)$ for knots with determinant 81

\begin{tabular}{|c|c|c|c|c|c|c|c|c|c|c|c|}
\hline $\mathrm{R}$ & $S$ & $\mathrm{~T}$ & knot & $\mathrm{R}$ & $S$ & $\mathrm{~T}$ & knot & $\mathrm{R}$ & $\mathrm{S}$ & $\mathrm{T}$ & knot \\
\hline-1 & 0 & -1 & \multirow{2}{*}{$11 \mathrm{a} 103$} & -2 & 0 & -1 & $13 n 403$ & 1 & -1 & -1 & $10_{99}$ \\
\hline 0 & 1 & 0 & & 2 & 0 & -1 & $13 n 405$ & -1 & 0 & 0 & $11 \mathrm{a} 58$ \\
\hline 0 & -1 & -1 & \multirow{2}{*}{$11 \mathrm{a} 165$} & 1 & 1 & 0 & $13 n 415$ & 1 & 0 & -1 & $12 \mathrm{n} 49$ \\
\hline-1 & 2 & 0 & & 2 & 0 & 0 & $13 n 440$ & 1 & -1 & 0 & $12 \mathrm{n} 440$ \\
\hline 0 & -1 & 0 & $11 \mathrm{a} 201$ & 0 & 2 & 0 & $13 n 1194$ & -2 & 0 & 0 & $13 n 436$ \\
\hline 1 & 0 & 0 & $12 \mathrm{n} 62$ & -1 & -1 & -1 & $13 n 1198$ & 1 & 1 & -1 & $13 n 447$ \\
\hline \multirow[t]{2}{*}{-2} & 1 & -1 & $12 \mathrm{n} 66$ & 1 & 0 & 1 & $13 n 1336$ & -1 & -1 & 0 & $13 \mathrm{n} 1156$ \\
\hline & & & & -1 & 0 & 1 & $13 \mathrm{n} 1680$ & 1 & -1 & 1 & $13 n 2199$ \\
\hline 0 & 0 & -1 & $3{ }_{1} \# 8_{11}$ & 0 & 1 & 1 & $13 n 1971$ & & & & \\
\hline 0 & 0 & 0 & $3_{1} \# 8_{10}$ & 0 & -1 & 1 & $13 n 3058$ & & & & \\
\hline
\end{tabular}

TABLE 3. Overview of knot types for the ribbon $\mathscr{B}(R, S, T)$ with determinant 81 . Knots in the right column are symmetric.

We interpret the fact that this family contains symmetric and possibly non-symmetric ribbon knots in the following way: the knots in the table cannot be transformed to a symmetric union in a way that their ribbon is respected. However, for some of them there exists a symmetric union presentation with another ribbon. Put another way, the knots in the right block in Table 3 correspond to the case 12 n268 in the previous section.

For $R=S=0$ the ribbon knots are composite and we obtain for $T=-3, \ldots, 3$ the knots $3_{1} \# 11$ n122, $3_{1} \# 10_{143}, 3_{1} \# 8_{11}, 3_{1} \# 8_{10}, 3_{1} \# 10_{147}, 3_{1} \# 11$ n106, $3_{1} \# 12 n 444$.

These composite ribbon knots consist of a trefoil and a knot concordant to the mirrored trefoil. Because of their striking non-symmetry, we think that they do not have symmetric union presentations and that proving this might be easiest among our candidates.

Conjecture 4.3. The (composite) knots $\mathscr{B}(0,0, T)$ are non-symmetric ribbon knots; less confident than for the determinant 9 cases: the knots 11a103, 11a165, 11a201, 12n62 and 12 n66 are non-symmetric ribbon knots. And, with specific ribbon: no knot $\mathscr{B}(R, S, T)$ can be transformed to a symmetric union respecting its ribbon. 
4.3. Non-symmetric ribbons for the remaining ribbon knots with determinant 225 . For the three knots $12 \mathrm{a} 348,12 \mathrm{a} 990$ and $12 \mathrm{a} 1225$ we found the two ribbons shown in Figure 8 Ribbon $\mathscr{C}$ yields for parameters $(S, T)=(0,1)$ the knot $12 \mathrm{a} 990$ and for $(2,0)$ the knot $12 \mathrm{a} 1225$. No knots with 13 crossings have been found for other parameters.

The knot $12 \mathrm{a} 348$ is obtained for the ribbon $\mathscr{D}$ with parameters $(-2,1,-1)$ and $(1,-2,0)$ and additional ribbon knots with 13 crossings are reported in Table 4 . For none of these a symmetric union presentation has been found.

\begin{tabular}{|r|r|r|l||r|r|r|l|}
\hline $\mathrm{R}$ & $\mathrm{S}$ & $\mathrm{T}$ & knot & $\mathrm{R}$ & $\mathrm{S}$ & $\mathrm{T}$ & knot \\
\hline-2 & 1 & -1 & \multirow{2}{*}{$12 \mathrm{a} 348$} & -1 & -1 & 0 & $13 \mathrm{a} 549$ \\
1 & -2 & 0 & & 0 & -1 & $13 \mathrm{a} 556$ \\
& & & & -2 & 0 & 0 & \} $13 \mathrm{a} 907$ \\
-2 & 0 & -1 & $13 \mathrm{a} 414$ & 1 & -1 & -1 & \} \\
0 & 0 & 0 & \multirow{2}{*}{$13 \mathrm{a} 521$} & 1 & -1 & 0 & $13 \mathrm{a} 1392$ \\
-1 & -1 & -1 & & & & \\
\hline
\end{tabular}

TABLE 4. Overview of knot types for the ribbon $\mathscr{D}(R, S, T)$.

It seems plausible to conjecture that the ribbons $\mathscr{C}$ and $\mathscr{D}$ generate only non-symmetric ribbon knots. Effective tools for tackling our conjectures do not yet exist, though.
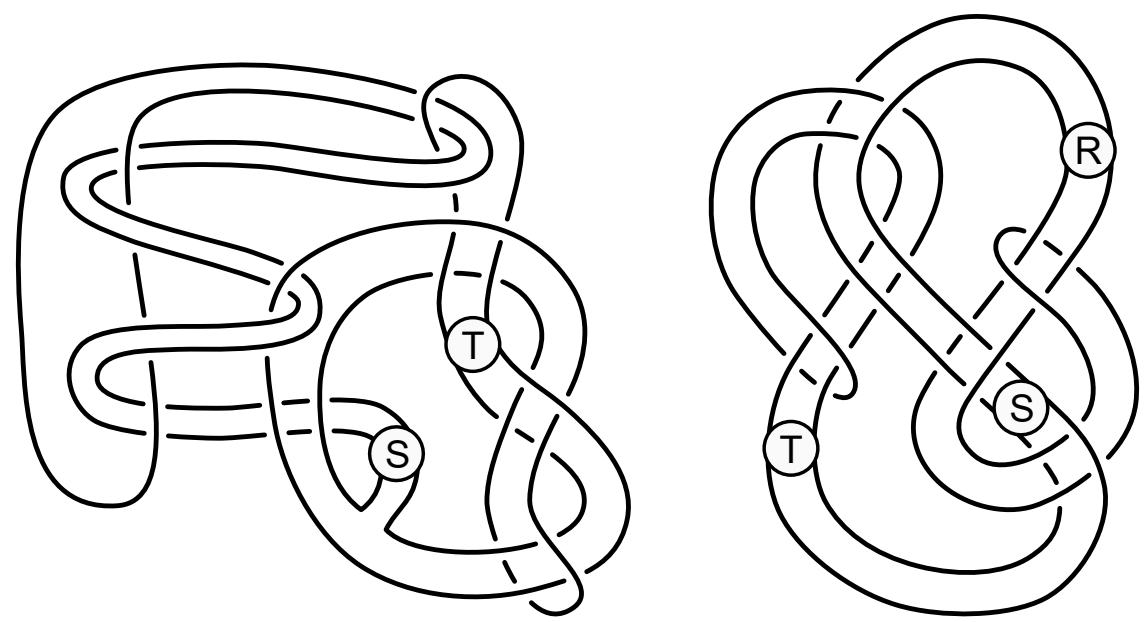

FIgurE 8. The ribbons $\mathscr{C}(S, T)$ and $\mathscr{D}(R, S, T)$ for knots with determinant 225 


\section{SYMMETRIC UNION DIAGRAMS AS BANDS WITHOUT CROSSINGS AND JUNCTIONS}

In this section we characterise bands constructed from symmetric unions as bands composed of a reduced set of elementary pieces. First, we introduce a normalised form for ribbon bands: Every ribbon band can be represented by the elementary pieces of Figure 9. see [Eisermann 09]. If we restrict this list of building blocks by omitting junctions and crossings, we obtain symmetric bands:
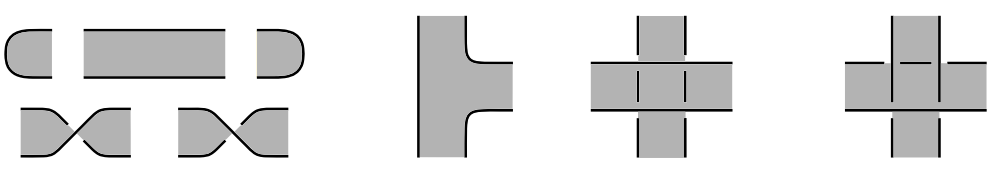

FIGURE 9. Elementary pieces of band diagrams: ends, strip, twists, junction, crossing, ribbon singularity

Proposition 5.1 (||Aceto 14|). A band can be constructed from a symmetric union diagram if and only if it has a band diagram composed of elementary pieces with neither crossings nor junctions.

One direction in the proof of this proposition is clear from an illustration as in Figure 1 . a slight modification of a symmetric band's projection on the symmetry plane has neither crossings nor junctions and only strips, ends, ribbon singularities and twists occur. Aceto observed that the other direction is true as well and obtained an estimate concerning the number of ribbon singularities in a specific family of ribbon knots.

We noticed earlier (see Figure 6 that for $S=-1,0$ the bands $\mathscr{A}(S, T)$ can be transformed to a symmetric band. Taking Proposition 5.1 into account, the symmetrisation of bands can be viewed as an elimination of crossings and junctions. For this, we provide two band moves in the following example.

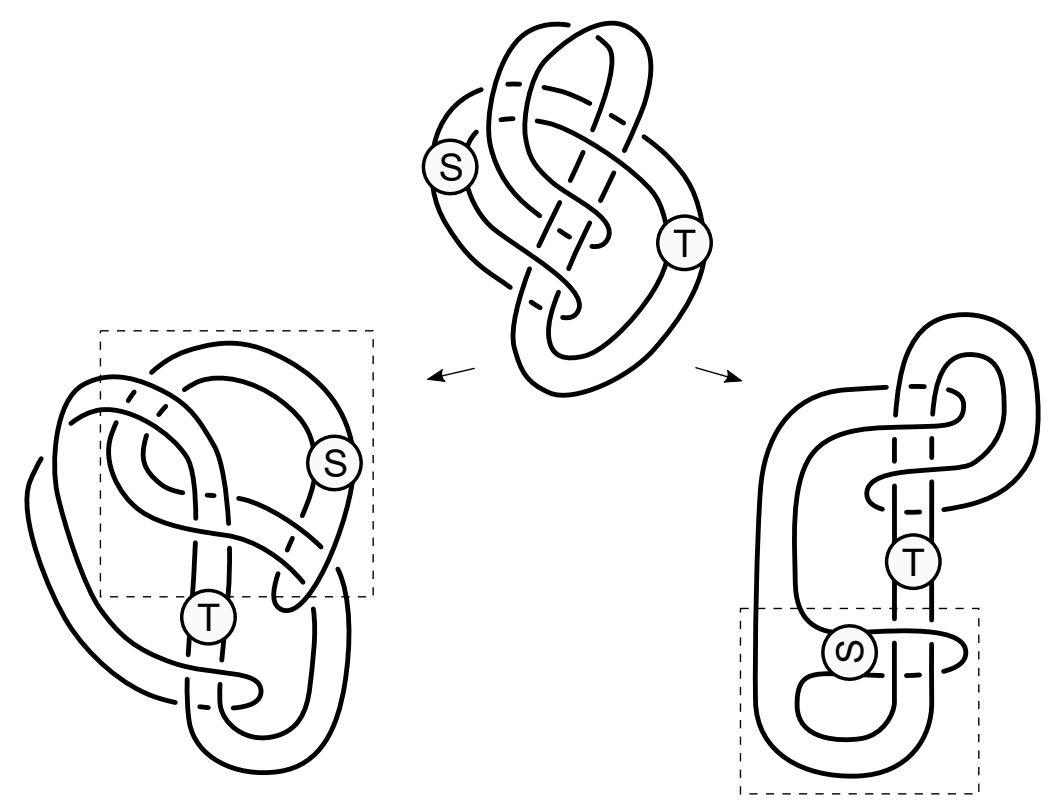

FIGURE 10. Preparation for the elimination of a crossing (left) or a junction (right) 


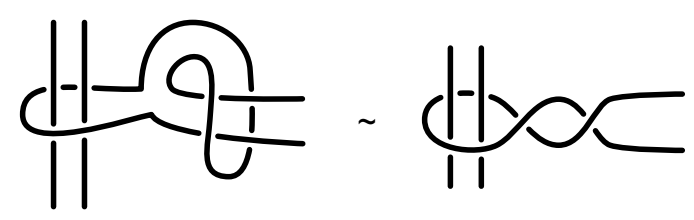

FIGURE 11. A band move useful for the elimination of a crossing

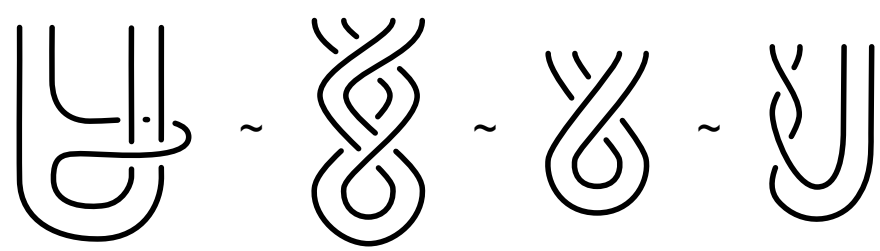

FIGURE 12. Removing a junction in $\mathscr{A}(-1, T)$ using Reidemeister 1 moves for bands

Example 5.2. We illustrate this again with the ribbon $\mathscr{A}(S, T)$. As the case $S=0$ is already shown in Figure 6, we now choose the case $S=-1$.

Elimination of a crossing: In the left side of Figure 10 the diagram is modified so that for $S=-1,0$ the crossing in the upper part can be removed. The move which eliminates this crossing for $S=-1$ is shown in Figure 11 .

Elimination of a junction: In the right side of Figure 10 we transform the diagram in a similar way as in Figure 6 and prepare the elimination of a junction. Reidemeister 1 type moves (with and without singularity) allow the removal of the junction, see Figure 12.

Note that these eliminations are not possible for $S \neq-1,0$.

Why, then, are the ribbons $\mathscr{B}(R, S, T)$ in Figure 7 not symmetric although they seem to be free of crossings and junctions? This is explained in Figure 13 . for convenience we allow an additional auxiliary elementary piece in the band construction. If this is expressed by elementary pieces, a junction or crossing is introduced, showing that the bands $\mathscr{B}(R, S, T)$ are not symmetric.

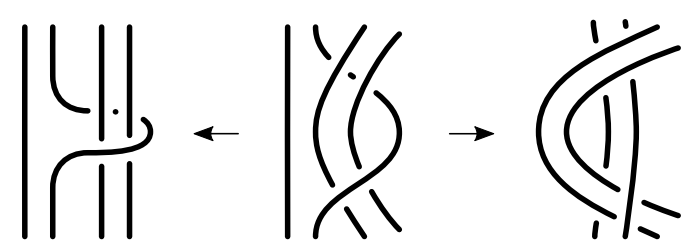

FIGURE 13. The auxiliary elementary piece in the middle transforms to a description with either a junction or a crossing if only elementary pieces are to be used.

The next step, a systematic study of band surfaces, their equivalence relations and invariants, is postponed to a future article. Suitable tools should then allow one, for instance, to show the equivalence or non-equivalence of the bands in Figure 14, all representing the knot 12n268. An analogous investigation for symmetric ribbons has been done in our articles [6] and [7]. 

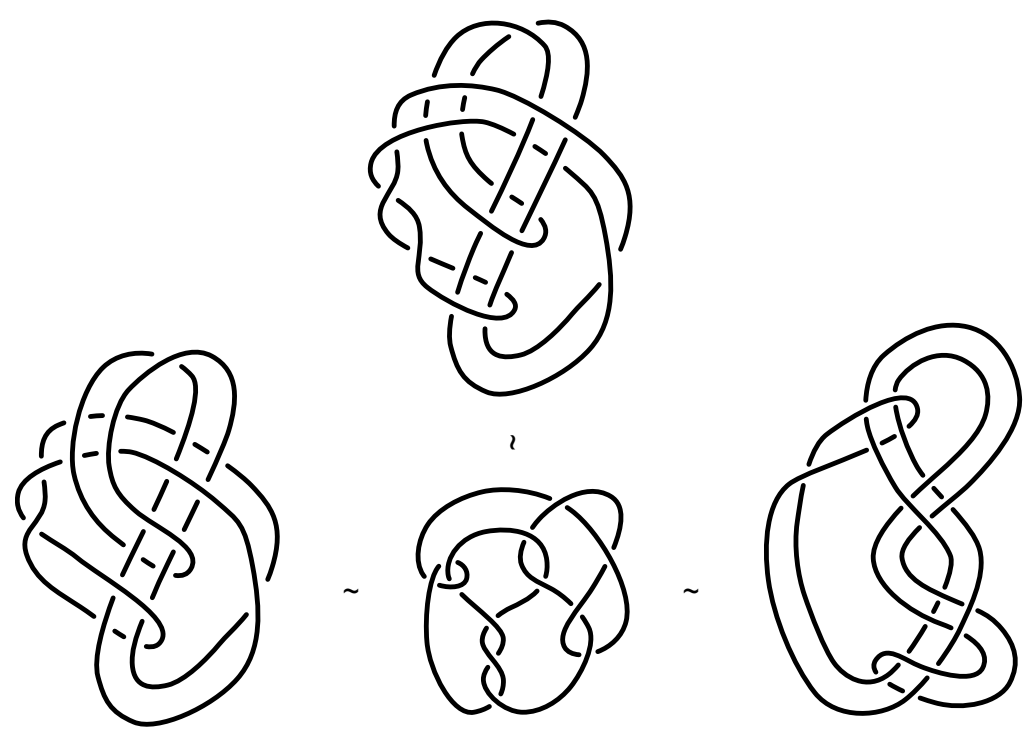

FIGURE 14. Three ribbons representing the knot 12n268 (shown in the middle): are the band diagrams $\mathscr{A}(2,-1)$ (left), $\mathscr{A}(-3,1)^{*}$ (top), and the diagram taken from template $3_{1}$, version $\mathrm{b}$ (right) equivalent? The left and top diagrams are related by mutation (Proposition 4.1) and the right diagram is symmetric.

\section{KNOTOID NOTATION FOR SYMMETRIC RIBBON DISKS}

This section contains the description of a new diagram type for bands constructed from symmetric unions. It can be seen as a continuation of Aceto's proposition in the last section and was already used in Figure 1 in the insets.

This notation is called knotoid diagram for a symmetric ribbon disk and is motivated by the observation that one half of a symmetric union diagram is redundant and that the height function, necessary to construct a band from a symmetric union diagram, can be taken directly from the diagram. This facilitates the construction and visualisation of symmetric bands.
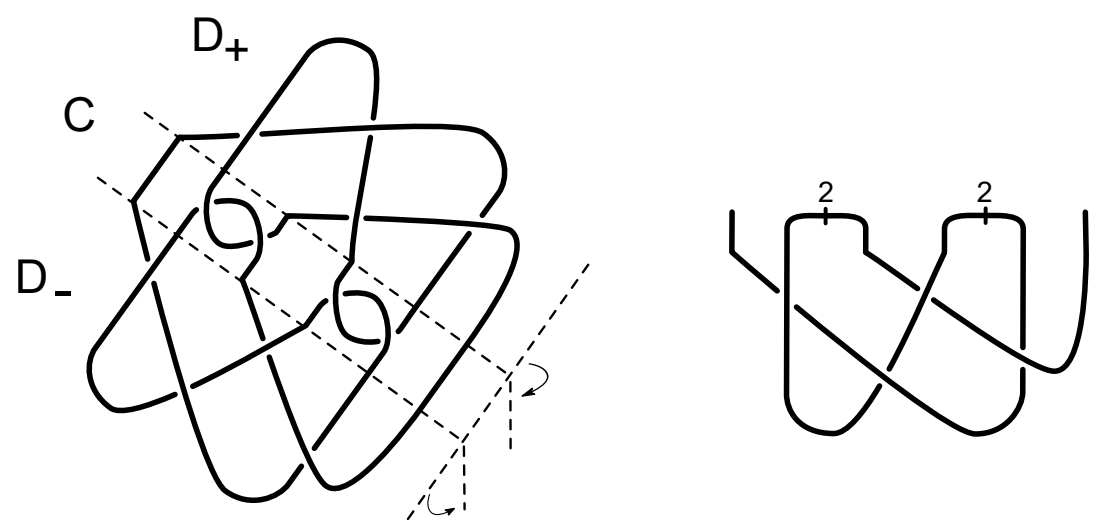

FIGURE 15. Illustration of the knotoid definition 
Definition 6.1 (knotoid diagram for a symmetric union). Consider a symmetric union diagram $D$, consisting of the two mirror-symmetric parts $D_{-}$and $D_{+}$and the central part $C$ as in Figure 15. The knotoid diagram for $D$ is the arc obtained from $D_{-}$by identifying the ends after cutting open the crossings on the axis. The (signed) numbers of crossings at the twist tangles in $C$ are used to decorate the arc so that the diagram $D$ can be recovered from its knotoid diagram. The knotoid endpoints correspond to the two perpendicular crossing points of the diagram with the axis. By folding the diagram down as indicated in the figure - using the diagram to supply the height function - we define the knotoid band given by the knotoid diagram of D. It is a ribbon of constant width except in the regions near crossings and is displayed in Figure 1 .

This is easily extended to the notion of knotoid diagram for a symmetric ribbon disk: We allow decorations with twist numbers for every segment between the arc's crossings and drop the requirement for the endpoint positions. In more detail, a knotoid diagram is a marked generically embedded $\operatorname{arc} A$ in the $(x, y)$-plane. It determines a 'vertical' immersion of $A \times[-1,1]$ in $\mathbb{R}^{3}$. The markings indicate how the immersion can be perturbed into a ribbon immersion. This step uses the over/under crossing information for choosing the ribbon singularities and the twist decorations for inserting twists. Note that it is not possible to construct a ribbon disk containing band crossings or junctions from a knotoid diagram.

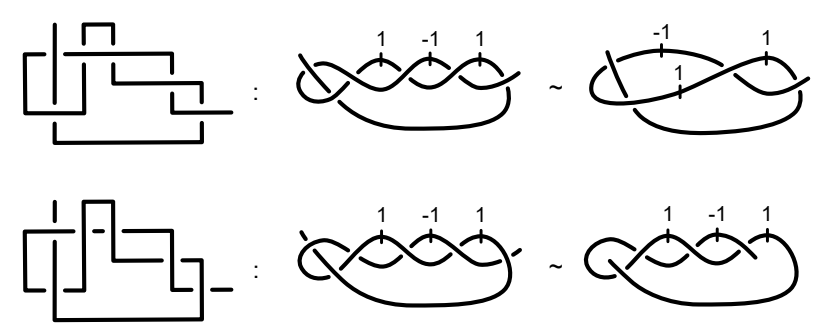

FIGURE 16. Knotoid diagrams for template $3_{1}$, version $\mathrm{b}$. The second and third columns show the knot $12 \mathrm{n} 268$.

We chose the name due to the similarity to knotoids due to Vladimir Turaev [Turaev 12]. Compare also the related definition of arc diagrams for ribbon 2-knots 1 , see [Kanenobu and Komatsu 17]. Knotoid diagrams and -bands enjoy the following properties:

a) Twist decorations on the same underpassing arc can be added because ribbon twists can be moved through singularities. This is not possible for overpassing arcs because the movement is prevented by the intersecting band.

b) Reidemeister moves for the knotoid diagram correspond to symmetric Reidemeister moves of the symmetric union diagram. They might be restricted by the arc's twist decorations. Analogously to the knotoid (and arc) diagram case the end of an arc can only be pulled out if it is an underpassing end. For an illustration see Figure 16 .

c) The insertion of twists in the band is easier for knotoid diagrams than for symmetric union diagrams: for the latter for arcs far away from the axis it is necessary to over- or undercross parts of the diagram. Knotoid diagrams avoid this because twists can be inserted everywhere.

Interestingly, knotoid bands for a knot given by a symmetric union diagram exist in two variants: if the diagram is rotated around its axis the diagram parts $D_{-}$and $D_{+}$are

\footnotetext{
${ }^{1}$ In the context of 2-dimensional knots the notion of 'symmetric ribbon spheres' was used by Takeshi Yajima [Yajima 64]. He proved that ribbon 2-knots can be deformed to a symmetric position in $\mathbb{R}^{4}$ (symmetric to the plane $x_{4}=0$ ). For comparison with classical knots we summarise the situation for 2-knots: all 2-knots are slice. There are slice 2-knots which are not ribbon. All ribbon 2-knots are symmetric (in the above sense).
} 
exchanged and the knotoid corresponding to $D_{+}$after rotation may yield a different band for the same knot, see Figure 18 (to use the rotated $D_{+}$and fold up is the same as to use $D_{-}$and fold down). Figure 16 contains two surprisingly different simplifications of the two bands for the knot 12 n268 obtained in this way.

In Figure 17 we explain the practical construction of a band from a knotoid diagram. In the same way a band for a complicated symmetric union can be constructed - via its knotoid diagram. We propose to study knotoid diagrams for symmetric ribbon disks in detail in a separate article.
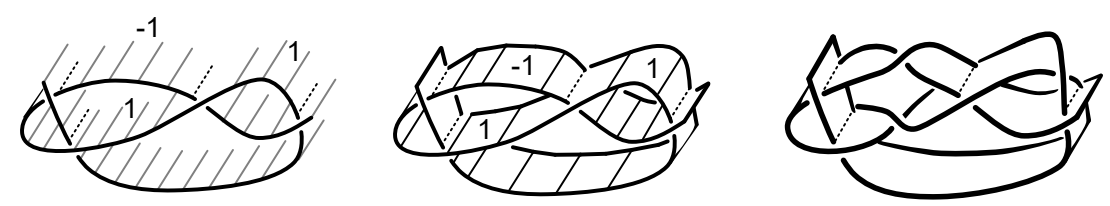

FIGURE 17. Practical steps for constructing the band from a knotoid diagram
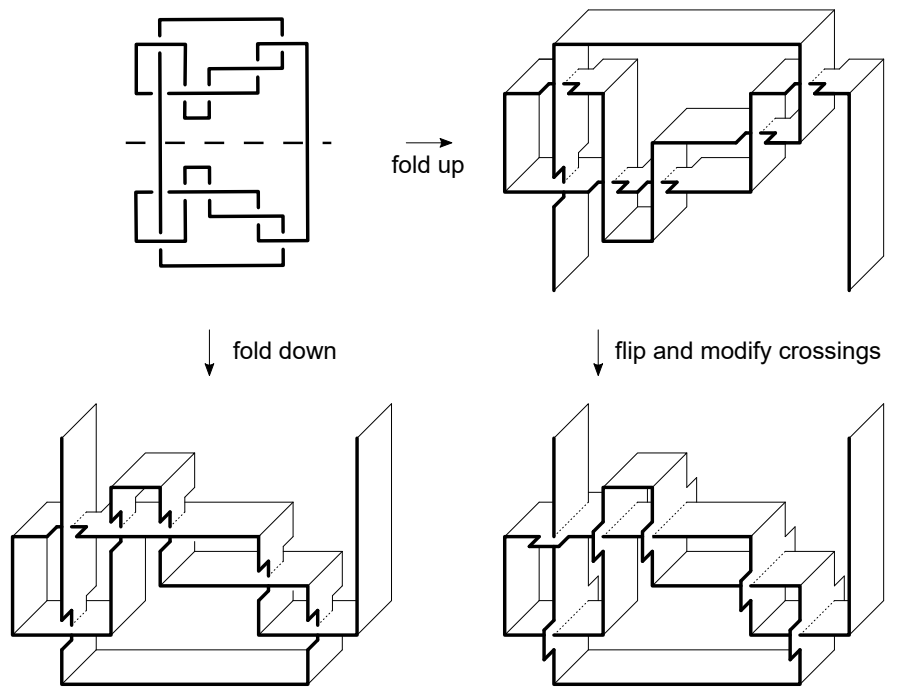

FIGURE 18. The two folding operations, illustrated with template $3_{1}$, version b

\section{OUTLOOK}

Besides proving that non-symmetric ribbon knots exist and pursuing the topic of band equivalences and corresponding invariants, an attractive goal is the study of ribbon knots with 13 crossings. For them Seeliger found 246 prime symmetric ribbon knots and an interesting discrepancy in the identification rate for different determinants: for instance, for determinant 289 there are 35 candidates and 32 symmetric hits, while for determinant 441 there are 23 candidates and no hits.

In another direction, a question similar to the slice-ribbon problem occurs: if we define symmetric slice knots by requiring a symmetric disk and allow twists at the saddles on the symmetry plane, can we prove that they are symmetric ribbon knots, i.e. can local minima be avoided? 


\section{ACKNOWLEDGEMENTS}

I thank Lukas Lewark and Johannes Renkl for commenting on earlier versions of this article and the referee for additional valuable help. The support of Axel Seeliger, who has left knot theory research to work in industry, is gratefully acknowledged.

\section{REFERENCES}

[Aceto 14] P. Aceto: Symmetric ribbon disks, J. Knot Theory Ramifications 23 (2014), 1450048

[Boocher, Daigle, Hoste and Zheng 09] A. Boocher, J. Daigle, J. Hoste and W. Zheng: Sampling Lissajous and Fourier Knots, Experiment. Math. 18 (2009), 481-497

[Cha and Livingston] J.C. Cha and C. Livingston: KnotInfo: Table of Knot Invariants, http://www.indiana.edu/ knotinfo

[Cha and Livingston 14] J.C. Cha and C. Livingston: Unknown values in the table of knots, preprint (last version 2014), arxiv:math.GT/0503125

[Eisermann 09] M. Eisermann: The Jones polynomial of ribbon links, Geometry \& Topology 13 (2009), 623-660

[Eisermann and Lamm 07] M. Eisermann and C. Lamm: Equivalence of symmetric union diagrams, J. Knot Theory Ramifications 16 (2007), 879-898

[Eisermann and Lamm 11] M. Eisermann and C. Lamm: A refined Jones polynomial for symmetric unions, Osaka J. Math. 48 (2011), 333-370

[Herald, Kirk and Livingston 10] C. Herald, P. Kirk and C.Livingston: Metabelian representations, twisted Alexander polynomials, knot slicing, and mutation, Math. Zeitschrift 265 (2010), 925-949

[Hoste, Thistlethwaite and Weeks 98] J. Hoste, M. Thistlethwaite and J. Weeks: The first 1,701,936 knots, Math. Intelligencer 20 (1998), 33-48

[Kanenobu 86] T. Kanenobu: Examples of polynomial invariants of knots and links, Math. Ann. 275 (1986), $555-572$

[Kanenobu 10] T. Kanenobu: Band surgery on knots and links, J. Knot Theory Ramifications 19 (2010), 15351547

[Kanenobu and Komatsu 17] T. Kanenobu and S. Komatsu: Enumeration of ribbon 2-knots presented by virtual arcs, J. Knot Theory Ramifications 26 (2017), 1750042

[Kearney 13] K. Kearney: The concordance genus of 11-crossing knots, J. Knot Theory Ramifications 22 (2013), 1350077

[Kinoshita and Terasaka 57] S. Kinoshita and H. Terasaka: On unions of knots, Osaka Math. J. 9 (1957), 131-153 [Knotscape 99] J. Hoste and M. Thistlethwaite: Knotscape - providing convenient access to tables of knots, http://www.math.utk.edu/ morwen/knotscape.html 1999

[Lamm 00] C. Lamm: Symmetric unions and ribbon knots, Osaka J. Math. 37 (2000), 537-550

[Lamm 06] C. Lamm: Symmetric union presentations for 2-bridge ribbon knots, preprint (2006), arxiv:math.GT/0602395

[Livingston 04] C. Livingston: The concordance genus of knots, Alg. \& Geom. Topology 4 (2004), 1-22

[Piccirillo 18] L Piccirillo: The Conway knot is not slice, preprint (2018), arxiv:math.GT/1808.02923

[Seeliger 14] A. Seeliger: Symmetrische Vereinigungen als Darstellungen von Bandknoten bis 14 Kreuzungen (Symmetric union presentations for ribbon knots up to 14 crossings), Diploma thesis, Stuttgart University (2014)

[Tanaka 10] T. Tanaka: Ribbon 2-knots with symmetric union presentations, J. Knot Theory Ramifications 19 (2010), 1-13

[Turaev 12] T. Turaev: Knotoids, Osaka J. Math. 49 (2012), 195-223

[Watson 07] L. Watson: Knots with identical Khovanov homology, Alg. \& Geom. Topology 7 (2007), 1389-1407

[Yajima 64] T. Yajima: On simply knotted spheres in $\mathbb{R}^{4}$, Osaka J. Math. 1 (1964), 133-152

\section{Christoph Lamm}

Rückertstr. 3, 65187 Wiesbaden

Germany

e-mail: christoph.lamm@web.de 
7. APPENDIX: THE 28 TEMPLATES GENERATING 122 SYMMETRIC UNION DIAGRAMS

The diagrams are grouped by determinant; further explanations are given in the text.

$\operatorname{det}=1$

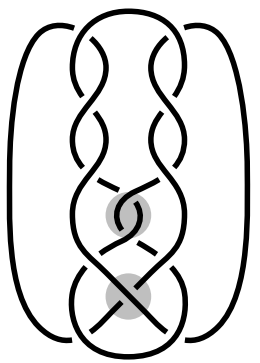

$K_{ \pm}=$triv, version a

$$
\begin{aligned}
& 11 \mathrm{n} 42=(2,0) \\
& 11 \mathrm{n} 49=(1,-1) \\
& 11 \mathrm{n} 116=(1,1) \\
& 12 \mathrm{n} 214=(3,0) \\
& 12 \mathrm{n} 309=(1,-2) \\
& \frac{12 \mathrm{n} 313}{12 \mathrm{n} 318}=(2,-1) \\
& 12 \mathrm{n} 430=(2,1)
\end{aligned}
$$

$\operatorname{det}=9$

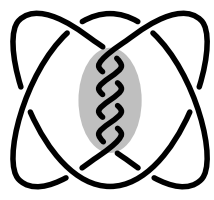

$K_{ \pm}=3_{1}$, version a $\underline{11 \ln 139}=(5)$ $12 \mathrm{n} 582=(6)$

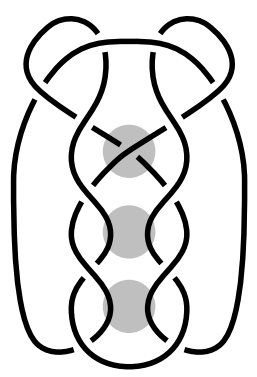

$K_{+}=3_{1}$, version b $12 \mathrm{n} 268=(1,-1,1)$ $\underline{12 \mathrm{n} 605}=(1,0,0)$

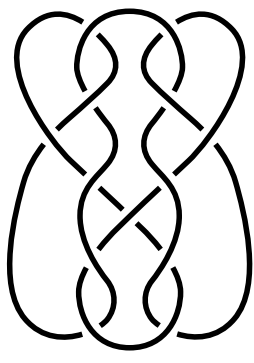

$K_{ \pm}=$triv, version b $12 \mathrm{n} 19=(1)$

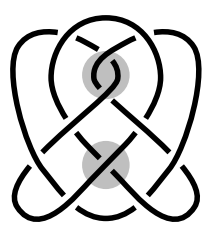

$K_{ \pm}=4_{1}$

$$
\begin{aligned}
& 11 \mathrm{n} 50=(3,0) \\
& \frac{11 \mathrm{n} 132}{12 \mathrm{n} 145}=(2,1) \\
& 12 \mathrm{n} 462=(2,-2) \\
& 12 \mathrm{n} 768=(3,1) \\
& 12 \mathrm{n} 838=(2,2)
\end{aligned}
$$

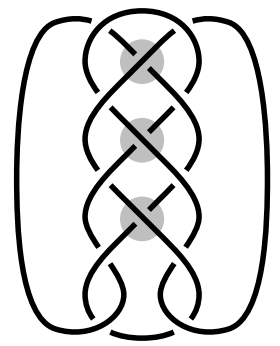

$K_{ \pm}=5_{1}$

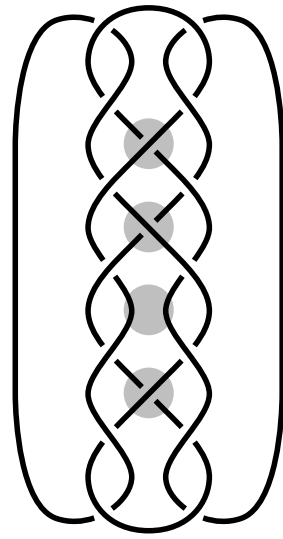

$K_{ \pm}=3_{1}$, version c

$$
\underline{12 \mathrm{n} 23}=(1,-1,0,1)
$$

$12 \mathrm{n} 676=(0,1,-3,1)$ 


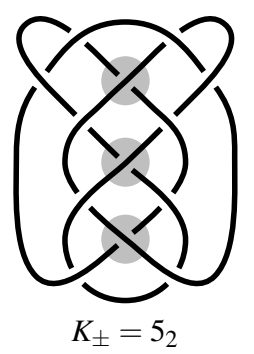

$$
\begin{aligned}
& \frac{11 \mathrm{n} 4}{11 \mathrm{n} 21}=(1,1,-1) \\
& 11 \mathrm{n} 83=(1,-1,0) \\
& 11 \mathrm{n} 172=(1,-1,3) \\
& 12 \mathrm{n} 24=(1,-1,-1) \\
& 12 \mathrm{n} 48=(2,0,-1) \\
& 12 \mathrm{n} 87=(0,2,0) \\
& 12 \mathrm{n} 288=(0,0,2) \\
& 12 \mathrm{n} 312=(0,1,1) \\
& 12 \mathrm{n} 360=(1,1,0) \\
& 12 \mathrm{n} 393=(1,-1,4) \\
& 12 \mathrm{n} 397=(2,1,-1) \\
& 12 \mathrm{n} 501=(2,0,0) \\
& 12 \mathrm{n} 708=(1,0,1) \\
& 12 \mathrm{n} 817=(1,0,-2)
\end{aligned}
$$
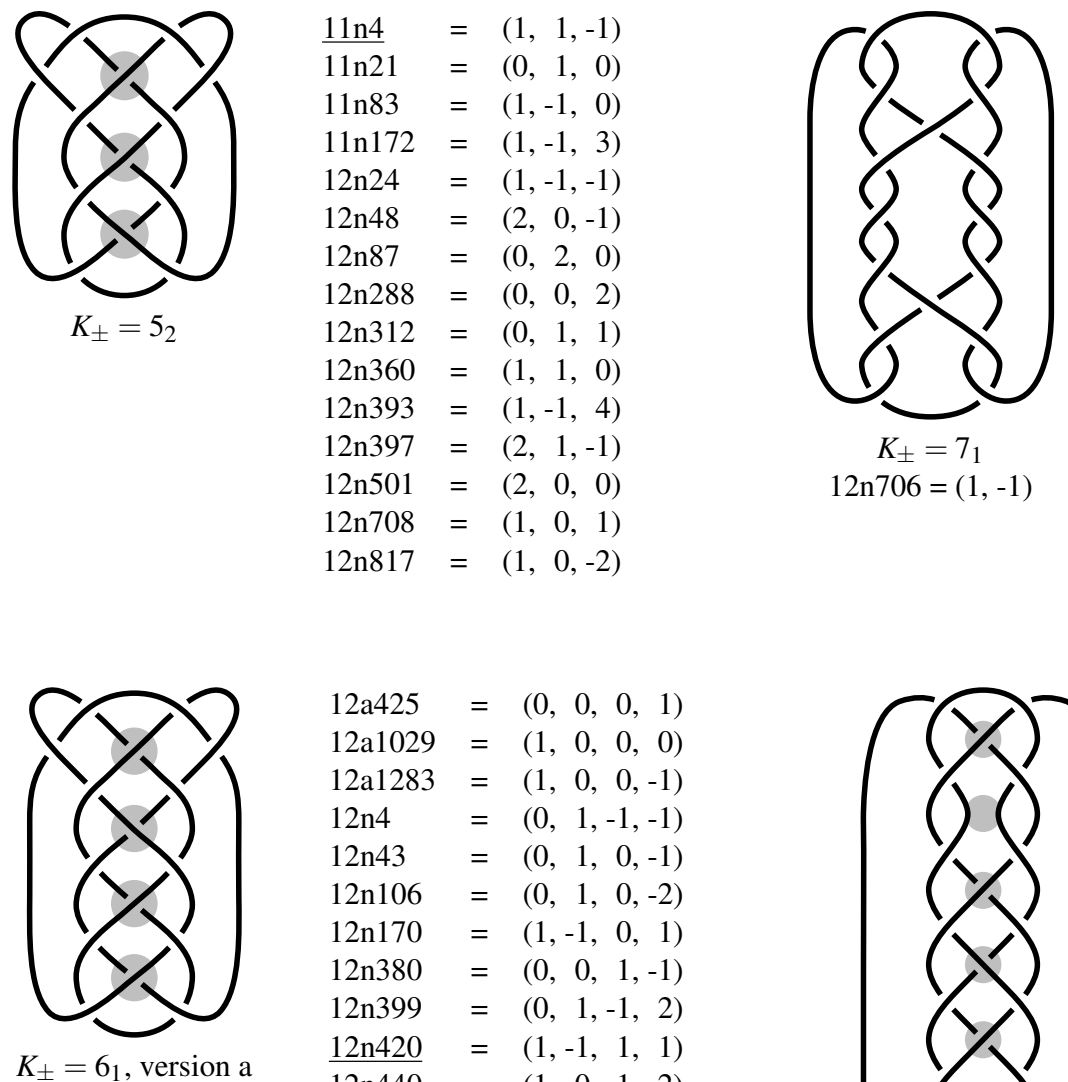

$$
\begin{aligned}
& 12 \mathrm{a} 425=(0,0,0,1) \\
& 12 \mathrm{a} 1029=(1,0,0,0) \\
& 12 \mathrm{a} 1283=(1,0,0,-1) \\
& 12 \mathrm{n} 4=(0,1,-1,-1) \\
& 12 \mathrm{n} 43=(0,1,0,-1) \\
& 12 \mathrm{n} 106=(0,1,0,-2) \\
& 12 \mathrm{n} 170=(1,-1,0,1) \\
& 12 \mathrm{n} 380=(0,0,1,-1) \\
& 12 \mathrm{n} 399=(0,1,-1,2) \\
& \underline{12 \mathrm{n} 420}=(1,-1,1,1) \\
& 12 \mathrm{n} 440=(1,0,1,-2) \\
& 12 \mathrm{n} 636=(2,-1,1,0) \\
& 12 \mathrm{n} 657=(1,0,-1,1) \\
& 12 \mathrm{n} 876=(1,-1,0,2)
\end{aligned}
$$

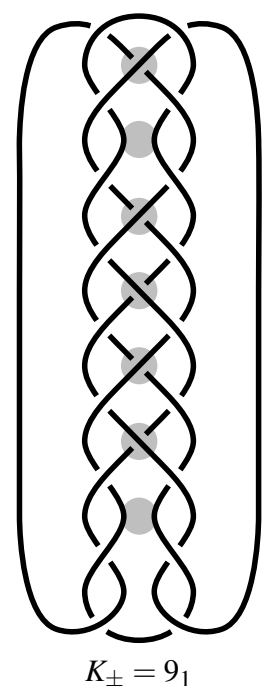

$11 \mathrm{a} 58=(0,0,1,-1,0,1,-1)$
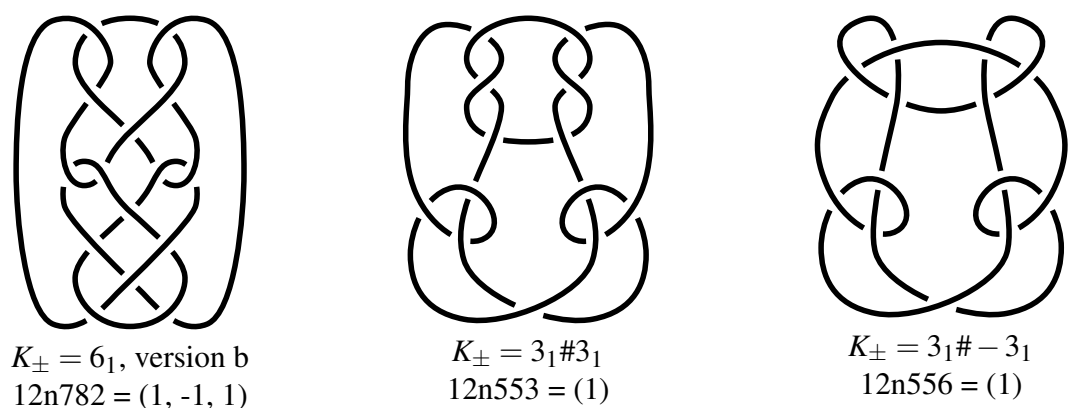


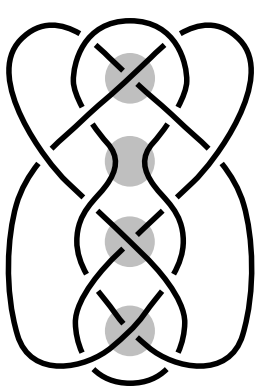

$K_{ \pm}=6_{2}$, version a

$$
\begin{array}{ll}
\frac{11 \mathrm{a} 28}{11 \mathrm{a} 35} & =(1,0,-1,1) \\
\text { 11a36 } & =(0,1,-1,1) \\
11 \mathrm{a} 87 & =(1,-1,1,-1) \\
11 \mathrm{a} 96 & =(0,0,-1,2) \\
\text { 11a115 } & =(0,1,-1,2) \\
11 \mathrm{a} 169 & =(1,0,-1,2) \\
\text { 11a316 } & =(1,-1,1,-2) \\
\text { 12a183 } & =(0,1,0,-1) \\
\text { 12a447 } & =(0,0,0,1) \\
\text { 12a667 } & =(0,1,0,0) \\
\text { 12a879 } & =(1,-1,0,1) \\
\text { 12a1011 } & =(1,0,0,-1) \\
\text { 12a1034 } & =(1,0,0,0) \\
\text { 12a1277 } & =(1,-1,0,0) \\
\text { 12n802 } & =(1,-1,-1,2)
\end{array}
$$

$\operatorname{det}=169$
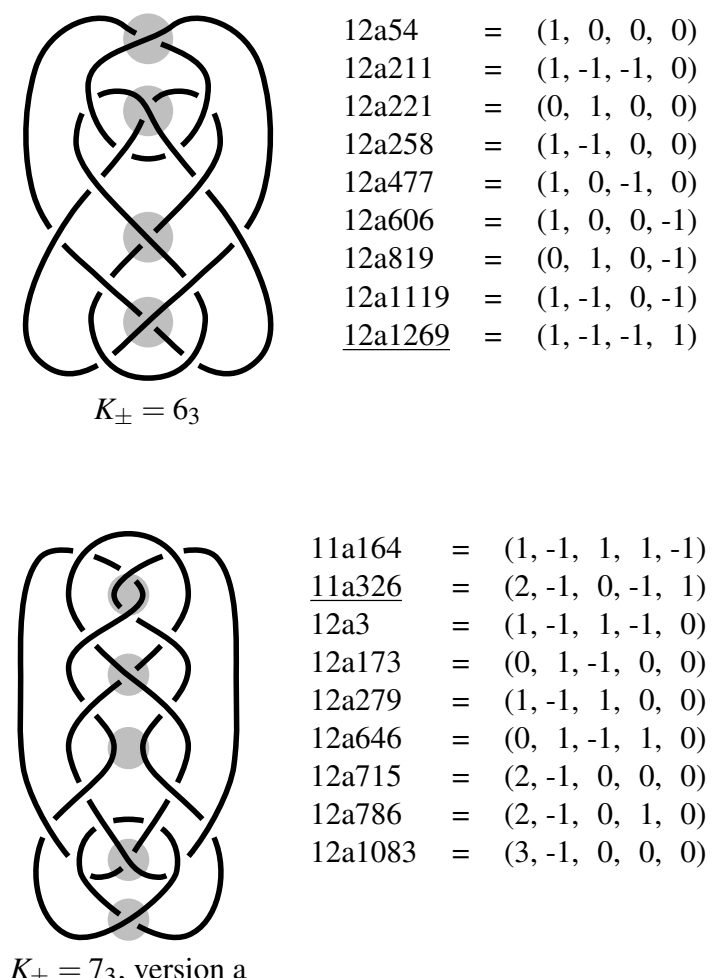

$$
\begin{aligned}
& 11 \mathrm{a} 164=(1,-1,1,1,-1) \\
& \underline{11 \mathrm{a} 326}=(2,-1,0,-1,1) \\
& \overline{12 \mathrm{a} 3}=(1,-1,1,-1,0) \\
& 12 \mathrm{a} 173=(0,1,-1,0,0) \\
& 12 \mathrm{a} 279=(1,-1,1,0,0) \\
& 12 \mathrm{a} 646=(0,1,-1,1,0) \\
& 12 \mathrm{a} 715=(2,-1,0,0,0) \\
& 12 \mathrm{a} 786=(2,-1,0,1,0) \\
& 12 \mathrm{a} 1083=(3,-1,0,0,0)
\end{aligned}
$$

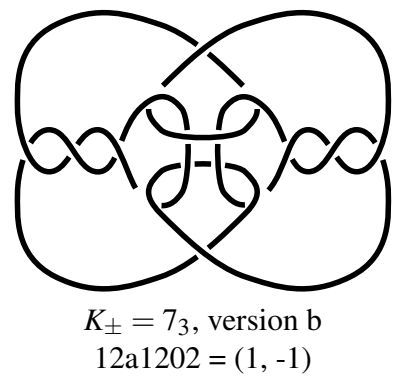

$K_{ \pm}=7_{3}$, version a

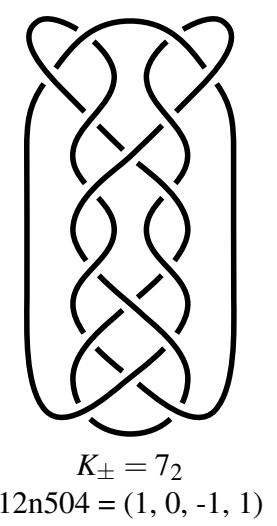
$K_{ \pm}=6_{2}$, version b
12 n702 $=(1,-1,1)$

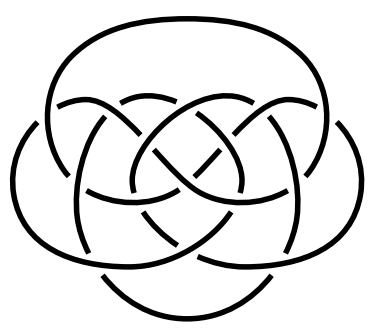

$K_{ \pm}=6_{2}$, version b 


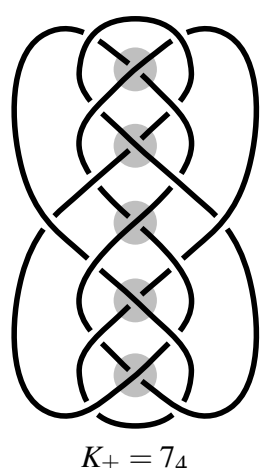

$\underline{12 \mathrm{a} 435}=(1,-1,1,-1,1)$

$12 \mathrm{a} 464=(2,-1,0,1,-1)$

$12 \mathrm{a} 975=(2,-1,1,-1,2)$

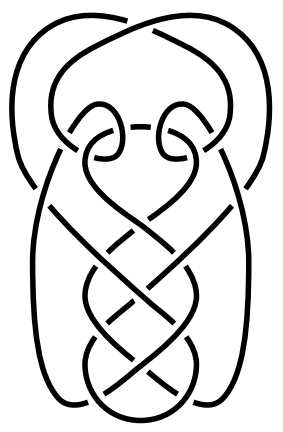

$K_{ \pm}=7_{5}$, version a

$12 \mathrm{a} 484=(1,-1,-1,1)$

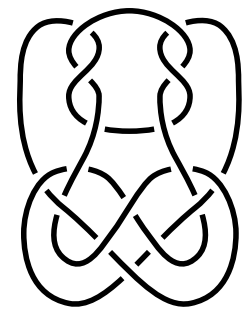

$K_{ \pm}=3_{1} \# 4_{1}$

$12 \mathrm{a} 427=(1,-1)$

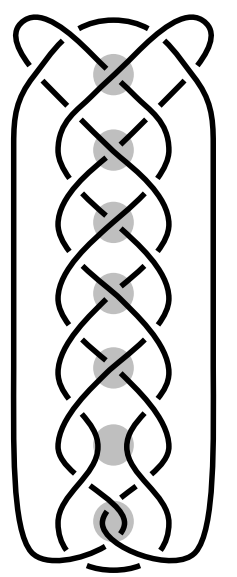

$K_{ \pm}=9_{2}$

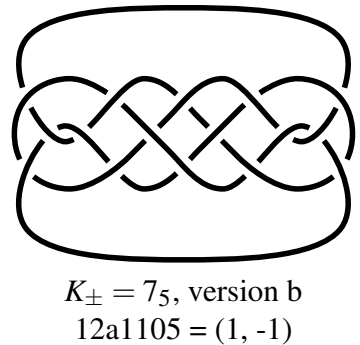

$12 \mathrm{a} 1105=(1,-1)$

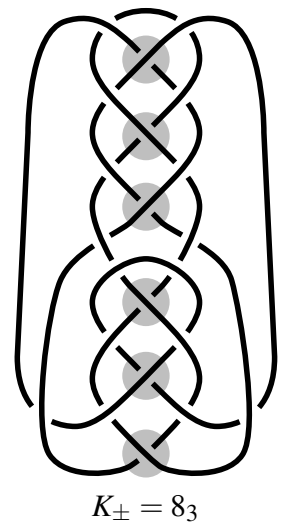

$\underline{12 \mathrm{a} 458}=(1,-1,1,-1,1,-1)$

$12 \mathrm{a} 473=(1,-1,1,2,-1,0)$

$12 \mathrm{a} 887=(2,-1,0,-2,1,0)$

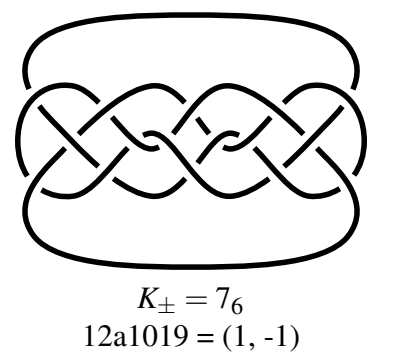

List of symmetric diagrams, page 4 (determinants 225, 289 and 361) 